\title{
Vortical and fundamental solitons in dipolar Bose-Einstein condensates trapped in isotropic and anisotropic nonlinear potentials
}

\author{
R. Kishor Kumar ${ }^{1}$, P. Muruganandam ${ }^{1}$ and B. A. Malomed ${ }^{2}$ \\ ${ }^{1}$ School of Physics, Bharathidasan University, Palkalaiperur Campus, Tiruchirappalli \\ 620024, Tamilnadu, India \\ ${ }^{2}$ Department of Physical Electronics, School of Electrical Engineering, Faculty of \\ Engineering, Tel Aviv University, Tel Aviv 69978, Israel
}

\begin{abstract}
We predict the existence of stable fundamental and vortical bright solitons in dipolar Bose-Einstein condensates (BECs) with repulsive dipole-dipole interactions (DDI). The condensate is trapped in the 2D plane with the help of the repulsive contact interactions whose local strength grows $\sim r^{4}$ from the center to periphery, while dipoles are oriented perpendicular to the self-trapping plane. The confinement in the perpendicular direction is provided by the usual harmonic-oscillator potential. The objective is to extend the recently induced concept of the self-trapping of bright solitons and solitary vortices in the pseudopotential, which is induced by the repulsive local nonlinearity with the strength growing from the center to periphery, to the case when the trapping mechanism competes with the long-range repulsive DDI. Another objective is to extend the analysis for elliptic vortices and solitons in an anisotropic nonlinear pseudopotential. Using the variational approximation (VA) and numerical simulations, we construct families of self-trapped modes with vorticities $\ell=0$ (fundamental solitons), $\ell=1$, and $\ell=2$. The fundamental solitons and vortices with $\ell=1$ exist up to respective critical values of the eccentricity of the anisotropic pseudopotential, being stable in the entire existence regions. The vortices with $\ell=2$ are stable solely in the isotropic model.
\end{abstract}




\section{Introduction}

Self-trapping of solitary modes, both fundamental and vortical ones, is one of basic topics in the current research work in the fields of nonlinear optics, matter-wave dynamics in quantum gases, and related areas [1, 2, 3, 4, 5, 6]. Usually, the self-trapping is provided by the self-focusing/self-attractive nonlinearity. Self-repulsion, if acting in the combination with periodic potentials, gives rise to localized modes of the bandgap type [4, 5]. Spatial modulation of the local nonlinearity may also help to support solitons [6], but it was assumed that the nonlinearity should be self-focusing on average in that case. Only very recently it has been demonstrated that purely repulsive nonlinear interactions may support bright solitons and solitary vortices, if the strength of the local cubic selfdefocusing term grows with the distance from the center $(r)$ faster than $r^{D}$, where $D$ is the spatial dimension [7]. In addition to straightforward realizations in optics, where the necessary spatial modulation of the local nonlinearity can be created by means of inhomogeneous doping [8], this setting may be realized in Bose and Fermi gases [9], making use of the Feshbach resonance (FR) controlled by spatially nonuniform magnetic [10], optical [11], or dc electric [12] fields. An extension of the setting for the model of an optical medium with the nonlocal self-defocusing thermal nonlinearity [13] has demonstrated that stable one-dimensional (1D) fundamental and dipole-mode solitons can be supported in this setting too, provided that the strength of the heating term grows with the coordinate faster than $|x|$ [14].

A particular topic in the studies of Bose-Einstein condensates (BECs), which has drawn a great deal of attention, is dealing with quantum gases of dipolar atoms and molecules [15]. Soliton modes were analyzed in models of dipolar BECs in various continuous [16] and discrete [17] settings (similar one-dimensional solitons were also predicted

in the model of the dipolar Tonks-Girardeau gas [22]). In this context, stable twodimensional (2D) solitons [18] and solitary vortices [19] were predicted in the isotropic form, assuming an inverted sign of the dipole-dipole interaction (DDI). Anisotropic fundamental 2D solitons were also found in a model based on the natural DDI sign [20, 21]. Numerical results for gap solitons in the dipolar BEC trapped in the 3D optical lattice have been reported too [23].

The objective of the present work is to consider the possibility of trapping the dipolar condensate in the plane of $(x, y)$, with the repulsive DDI between dipole moments polarized in the perpendicular direction, along axis $z$. The trapping is provided by the repulsive contact interaction, whose strength is assumed to grow $\sim r^{4}$ in the horizontal plane, and by the usual linear harmonic-oscillator (HO) potential applied in the vertical direction.

If the DDI is provided by atomic or molecular magnetic moments, the assumed spatial modulation of the contact self-repulsion should be imposed by the FR controlled by the optical or electric field, as the external magnetic field serves to polarize the magnetic moments. The power-law growth of the repulsion strength does not imply 
that the respective control field must grow similarly. Instead, it is enough to provide a weak transverse modulation of the field, which gradually reduces the local detuning of the FR with the increase of $r$, on the characteristic scale of $r \sim 100 \mu \mathrm{m}$ [7]. Such a weak modulation can be easily implemented by making the laser beam slightly focusing or defocusing, or applying the dc electric field induced by the capacitor with slightly nonflat electrodes. If, on the other hand, the DDI is provided by molecular electric dipoles, they must be polarized by means of an external electrostatic field, while a magnetic or optical field may be used to control the local detuning of the FR. In particular, a weakly nonuniform magnetic field may be applied by means of a tapered solenoid [24] or appropriately designed magnetic lattice [25].

In this work, we consider both isotropic and anisotropic shapes of the effective nonlinear pseudopotential acting in the $(x, y)$ plane. It is relevant to mention that the existence and stability of elliptic vortices in anisotropic HO traps was previously studied for the self-repulsive [27] and attractive [28] signs of the local cubic nonlinearity, but anisotropic nonlinear pseudopotentials were not considered before in any form, to the best of our knowledge.

The analysis is based on the variational approximation (VA) and direct simulations in imaginary and real time. The VA provides an initial approximation for the imaginarytime integration (it is known that this method is relevant for constructing both fundamental and vortical stationary modes [26]), while the stability of the stationary modes is tested by means of the real-time propagation. We aim to construct vortical modes with topological charges $\ell=1$ and 2 , as well as fundamental solitons with $\ell=0$.

The paper is structured as follows. The model and VA are introduced in Section II. The results for the vortices and fundamental solitons are reported in Sections III and IV, respectively. In particular, we find that the modes with $\ell=1$ and 0 are stable in their full existence domains, up to the largest eccentricity which admits the existence of the trapped modes in the anisotropic nonlinear pseudopotential, while the double vortices with $\ell=2$ are stable only in the isotropic pseudopotential. The paper is concluded by Section V.

\section{The mean-field description}

In the mean-field approximation, a dipolar BEC with $N$ atoms of mass $m$ at zero temperature is described by the Gross-Pitaevskii equation (GPE),

$$
\begin{aligned}
& i \frac{\partial \psi(\mathbf{r}, t)}{\partial t}=\left[-\frac{1}{2} \nabla^{2}+\frac{1}{2} z^{2}+g V_{\mathrm{NL}}(\mathbf{r})|\psi(\mathbf{r}, t)|^{2}\right. \\
& \left.+g_{\mathrm{d}} \int U_{\mathrm{DD}}\left(\mathbf{r}-\mathbf{r}^{\prime}\right)\left|\psi\left(\mathbf{r}^{\prime}, t\right)\right|^{2} d \mathbf{r}^{\prime}\right] \psi(\mathbf{r}, t),
\end{aligned}
$$

where $\psi(\mathbf{r}, t)$ is the mean-field wave function subject to the normalization condition,

$$
\int|\psi(\mathbf{r}, t)|^{2} d \mathbf{r}=1
$$


and the kernel of the DDI is $U_{\mathrm{DD}}(\mathbf{r})=\left(1-3 \cos ^{2} \theta\right) r^{-3}$, where $\theta$ is the angle between $\mathbf{r}$ and the direction of the polarization of the dipoles, $z$. In this direction, the condensate is confined by the HO potential with trapping frequency $\omega_{z}$. Accordingly, in equation (1) lengths are measured in units of the respective HO length, $l_{z} \equiv \sqrt{\hbar / m \omega_{z}}$, frequency in units of $\omega_{z}$, and time $t$ in units of $\omega_{z}^{-1}$, the contact-interaction and DDI strengths being, respectively, $g=4 \pi a_{s} N / l_{z}$ and $g_{\mathrm{d}}=N d^{2} m /\left(\hbar^{2} l_{z}\right)$, where $a_{s}$ is the scattering length of atomic collisions, and $d$ is the magnetic moment.

As said above, the repulsive contact interactions are subject to the modulation in the $(x, y)$ plane, with local strength growing from the center to periphery,

$$
V_{\mathrm{NL}}=\left(1+\epsilon_{1} x^{2}+\epsilon_{2} y^{2}\right)^{2}
$$

which corresponds to the action of an effective nonlinear quartic potential. As said above, in the $2 \mathrm{D}$ space we need to introduce the nonlinearity-strength-modulation function growing faster than $r^{D} \equiv r^{2}$, therefore we here adopt the quartic radial profile. Generally, the pseudopotential corresponding to the azimuthal modulation profile in (3) is anisotropic, with eccentricity

$$
\delta \equiv \frac{\epsilon_{2}-\epsilon_{1}}{\epsilon_{1}+\epsilon_{2}} .
$$

\section{Vortex solitons}

\subsection{The energy minimization}

In terms of cylindrical coordinates $z, \rho$, and $\phi$, stationary solutions to Eq. (1) for vortices with integer topological charge $\ell \geq 0$ are looked for in the usual form,

$$
\psi_{\ell}=\exp (-i \mu t+i \ell \phi) \rho^{\ell} U_{\ell}(\rho, z, \phi),
$$

where function $U_{\ell}$ remains finite at $\rho=0$, and decays as $\rho^{\ell}\left|U_{\ell}\right| \approx \sqrt{\mu / g}\left(\epsilon_{1} x^{2}+\epsilon_{2} y^{2}\right)^{-1}$ at $\rho \rightarrow \infty$, according to the Thomas-Fermi approximation [7]. The dependence on angular coordinate $\phi$ in this function is present in the case of anisotropic modulation pattern (3). In the latter case, $U_{\ell}$ is complex, while it is real for the isotropic setting.

The energy functional corresponding to GPE (1) is

$$
\begin{aligned}
& E=\frac{1}{2} \int\left[|\nabla \psi|^{2}+z^{2}|\psi|^{2}+g V_{\mathrm{NL}}(\mathbf{r})|\psi(\mathbf{r}, t)|^{4}\right. \\
& \left.+g_{\mathrm{d}} \int U_{\mathrm{DD}}\left(\mathbf{r}-\mathbf{r}^{\prime}\right)|\psi(\mathbf{r}, t)|^{2}\left|\psi\left(\mathbf{r}^{\prime}, t\right)\right|^{2} d \mathbf{r}^{\prime}\right] d \mathbf{r} .
\end{aligned}
$$

To predict vortex-soliton modes (5) by means of the VA, we calculate the value of functional (1), using expression (5) with a real Gaussian ansatz for $U_{\ell}(\rho, z)$, cf. Ref. [19]:

$$
U_{\ell}(\rho, z)=A_{\ell} \exp \left[-\frac{1}{2}\left(\alpha \rho^{2}+\gamma z^{2}\right)\right],
$$

where $\alpha$ and $\rho$ are free variational parameters, and the amplitude is determined by normalization condition (2):

$$
A_{\ell}=\pi^{-3 / 4}(\ell !)^{-1 / 2} \sqrt{\alpha^{\ell+1}} \gamma^{1 / 4} .
$$


Note that, even in the case of the anisotropic modulation profile (3), the isotropic form is adopted for ansatz (7), as otherwise the application of the VA to vortices is too cumbersome. The results for $\ell=1$ and 2 are

$$
\begin{aligned}
& E_{1}=\alpha+\frac{1}{4}\left(\gamma+\frac{1}{\gamma}\right)+\frac{1}{2} \sqrt{\frac{\gamma}{2 \pi}}\left[\frac{g}{32 \pi}\right. \\
& \left.\times \frac{9 \epsilon_{1}^{2}+9 \epsilon_{2}^{2}+12 \epsilon_{2} \alpha+8 \alpha^{2}+6 \epsilon_{1}\left(\epsilon_{2}+2 \alpha\right)}{\alpha}+\frac{g_{\mathrm{d}}}{3} \alpha f_{1}(\kappa)\right], \\
& E_{2}=\frac{3}{2} \alpha+\frac{1}{4}\left(\gamma+\frac{1}{\gamma}\right)+\frac{3}{8} \sqrt{\frac{\gamma}{2 \pi}}\left[\frac{g}{64 \pi}\right. \\
& \left.\times \frac{15\left(3 \epsilon_{1}^{2}+2 \epsilon_{1} \epsilon_{2}+3 \epsilon_{2}^{2}\right)+40\left(\epsilon_{1}+\epsilon_{2}\right) \alpha+16 \alpha^{2}}{\alpha}+\frac{g_{\mathrm{d}}}{3} \alpha f_{2}(\kappa)\right],
\end{aligned}
$$

where $\kappa \equiv \sqrt{\gamma / \alpha}$ is the aspect ratio of ansatz (7), and the following ad hoc functions are defined:

$$
\begin{aligned}
& f_{1}(\kappa) \equiv-1+3 \int_{0}^{1} R(\kappa, x)\left[1+Q^{2}(\kappa, x)\right] d x \\
& f_{2}(\kappa) \equiv-1+3 \int_{0}^{1} R(\kappa, x)\left[1+\frac{2}{3} Q^{2}(\kappa, x)+Q^{4}(\kappa, x)\right] d x \\
& R(\kappa, x) \equiv \frac{(\kappa x)^{2}}{(\kappa x)^{2}+\left(1-x^{2}\right)}, Q(\kappa, x) \equiv \frac{1-x^{2}}{(\kappa x)^{2}+\left(1-x^{2}\right)}
\end{aligned}
$$

\subsection{Vortices with $\ell=1$}

As seen from contour plots of the energy displayed in figures 1(a) and 1(b) for $\ell=1$, in both the isotropic and anisotropic versions of the model the energy, considered as a function of the variational parameters, has a well-defined minimum, which should correspond to a (presumably stable) stationary vortex soliton. Values of the parameters corresponding to this solution were found from a numerical solution of the equations defining the energy minimum:

$$
\frac{\partial E}{\partial \alpha}=\frac{\partial E}{\partial \gamma}=0
$$

Next, the vortex-soliton profiles predicted by the VA were used as initial conditions to generate their numerically exact counterparts by means of the imaginarytime integration method, in the isotropic and anisotropic models alike. Numerical simulations, in the imaginary and real time alike, were carried out using a method combining the split-step Crank-Nicolson algorithm and fast Fourier transform [29].

The results demonstrate that a difference $\lesssim 10 \%$ between the VA-predicted and numerically generated vortex profiles. The numerically constructed families of the vortex solitons in the isotropic and anisotropic systems are illustrated, respectively, in figures 2 and 3 by plots showing the dependence of the chemical potential [see equation (5)] and root-mean-square (rms) radius of the vortices on strength $g$ of the local repulsion, at several constant values of the long-range repulsion strength, $g_{\mathrm{d}}$, including $g_{\mathrm{d}}=0$, 

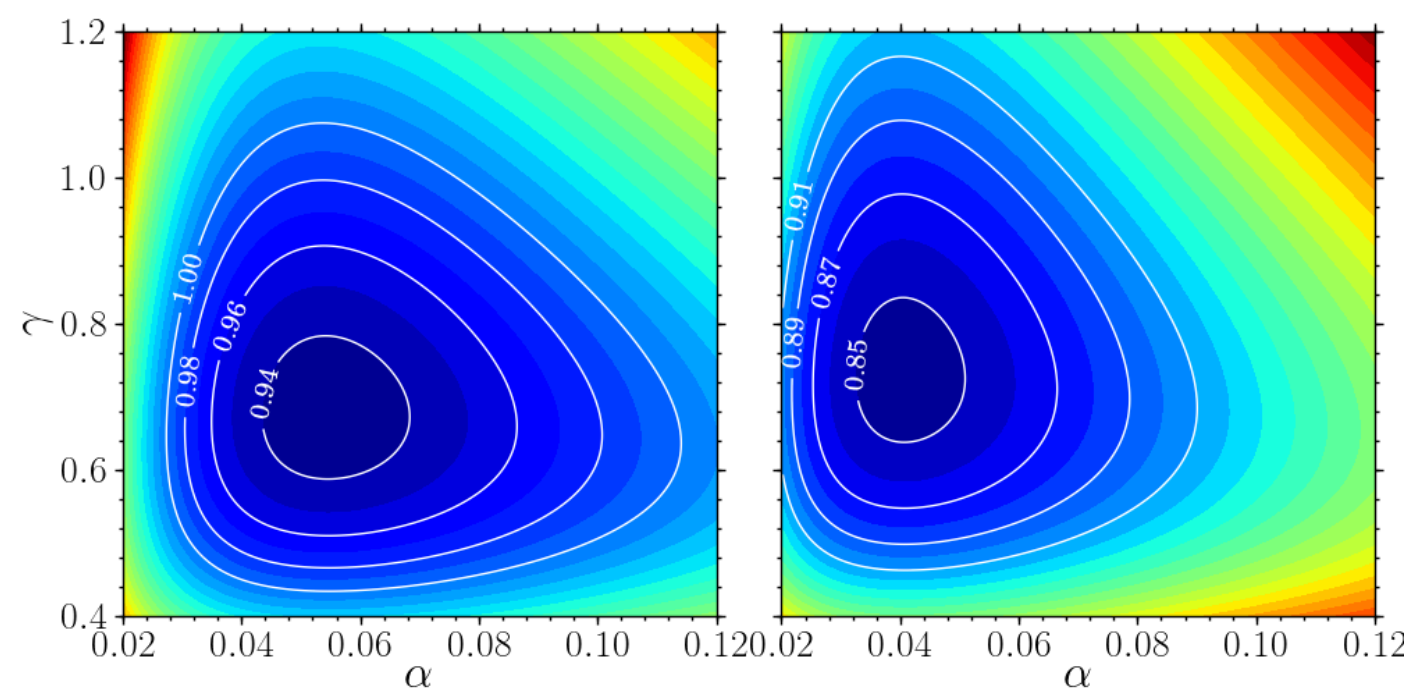

Figure 1. (Color online) GPE energy functional (6) for the vortex-soliton ansatz (7) with $\ell=1$ as a function of variational parameters $\alpha$ and $\gamma$. The interaction strengths are $g=20, g_{d}=30$, and parameters of the quartic isotropic and anisotropic nonlinear potential (3) are $\epsilon_{1}=\epsilon_{2}=0.1$ in (a), and $\epsilon_{1}=0.05, \epsilon_{2}=0.1$ in (b).

for the sake of the comparison with the usual model which does not include the DDI. The decrease of the radius with the increase of $g$, observed in figures 2(b) and 3(b), is a natural property of solitons supported by the growing repulsive nonlinearity [7]. It is natural too that the radius increases with the strength of the additional repulsive DDI, which is seen in the same plots.
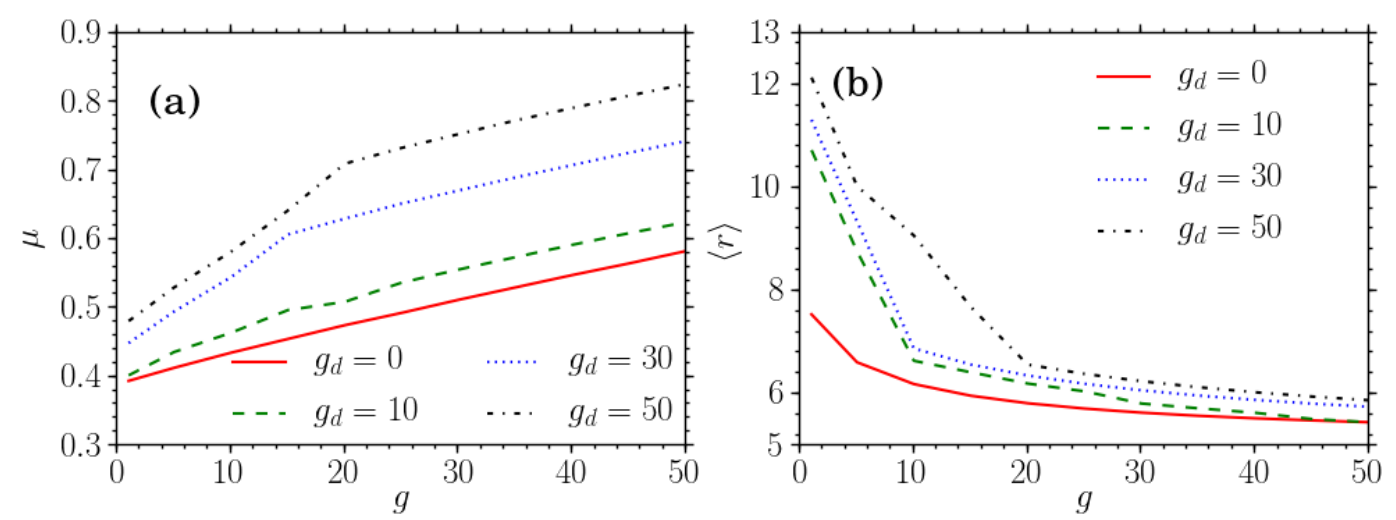

Figure 2. (Color online) The numerically calculated chemical potential (a), and the rms radius (b), as functions of the contact-interaction strength, at fixed values of the DDI strength, for vortex solitons with topological charge $\ell=1$ trapped in the isotropic nonlinear pseudopotential with $\epsilon_{1}=\epsilon_{2}=0.1$.

In the anisotropic model, it has been found that the vortex solitons with $\ell=1$ exist when eccentricity (4) takes values below a certain critical value, $\delta_{\text {cr }}$, which may be 

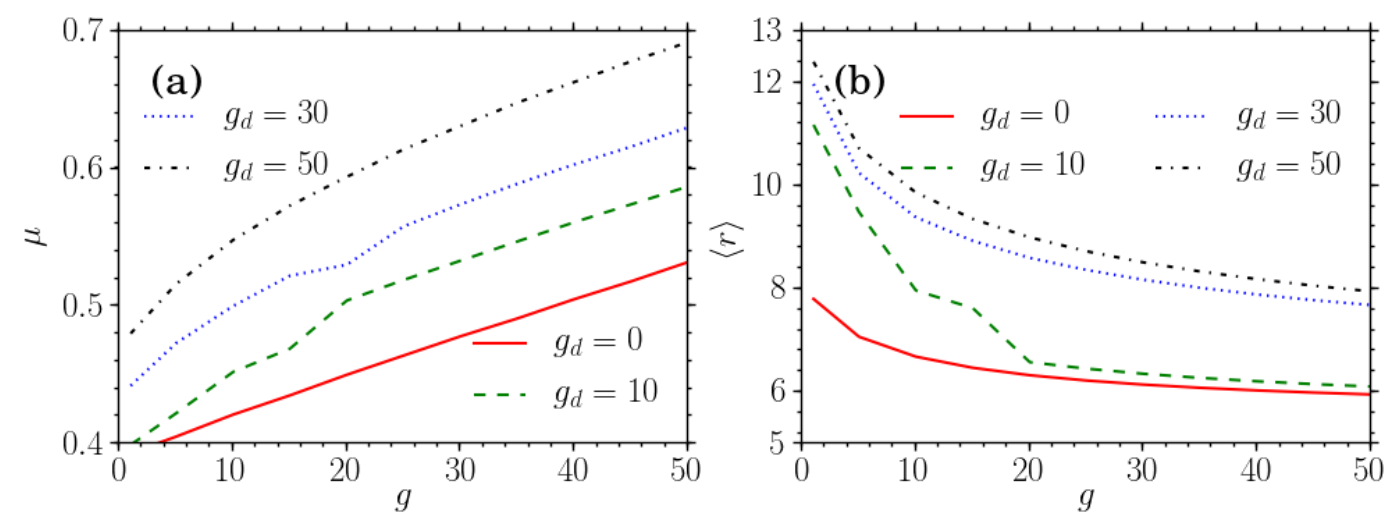

Figure 3. (Color online) The same as in figure 2, but for elliptic vortices trapped in the anisotropic pseudopotential with $\epsilon_{1}=0.05, \epsilon_{2}=0.1$.

quite close to 1, i.e., the critical shape, beyond which the vortex does not exist, may be strongly elongated. In particular,

$$
\left(\delta_{\mathrm{cr}}\right)_{\ell=1}=0.923
$$

was found for the elliptic vortex with $g=20$ and $g_{\mathrm{d}}=30$. A systematic dependence of the critical eccentricity on the DDI strength is presented by the plot in figure 4. The stronger isotropic DDI naturally tends to suppress the ellipticity, which explains the gradual decrease of $\delta_{\text {cr }}$ with the increase of $g_{\mathrm{d}}$.

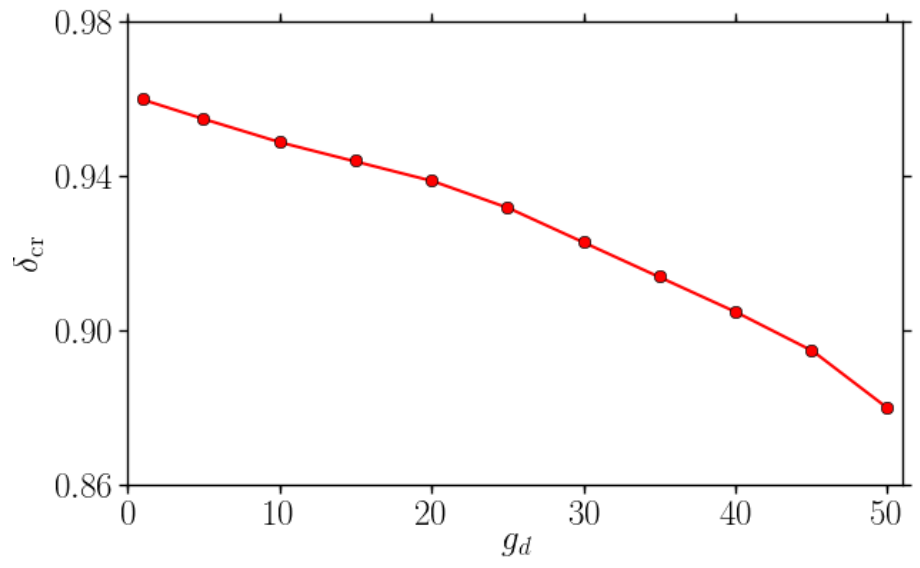

Figure 4. (Color online) The critical eccentricity for the existence of elliptic vortices with $\ell=1$, trapped in the anisotropic pseudopotential with $g=20$, as a function of the strength of the dipole-dipole repulsion.

Then, the stability of the so generated modes was tested by means of simulations of their perturbed evolution in real time. The conclusion is that all the solitary vortices with $\ell=1$ are stable in the isotropic and anisotropic settings alike, see examples of the evolution in figures 5 and 6, respectively (in fact, these figures also display typical shapes of the isotropic and anisotropic vortices). In this connection, it is relevant to 
mention that the increase of the chemical potential with $g$, which is observed in figures 2(a) and 3(a), implies that the vortex families satisfy the "anti-Vakhitov-Kolokolov" criterion, which plays the role of the necessary stability condition for localized modes supported by repulsive nonlinearities [30].
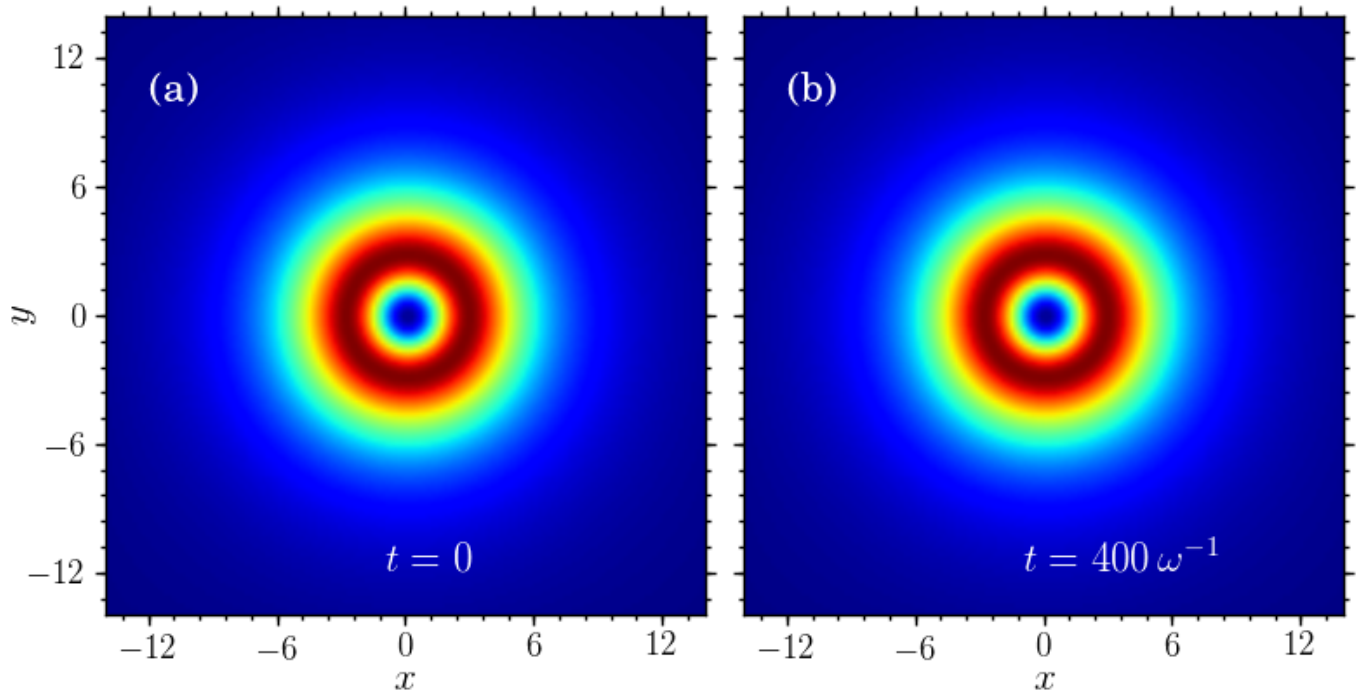

Figure 5. (Color online) An example of stable evolution of the vortex soliton with $\ell=1$ in the isotropic system. Shown are the initial state, obtained by means of the imaginary-time propagation, starting from Gaussian ansatz (7) with $\alpha=0.05412$ and $\gamma=0.6798$, and the final result of subsequent real-time simulations. The parameters are $g=20, g_{d}=30$, and $\epsilon_{1}=\epsilon_{2}=0.1$.
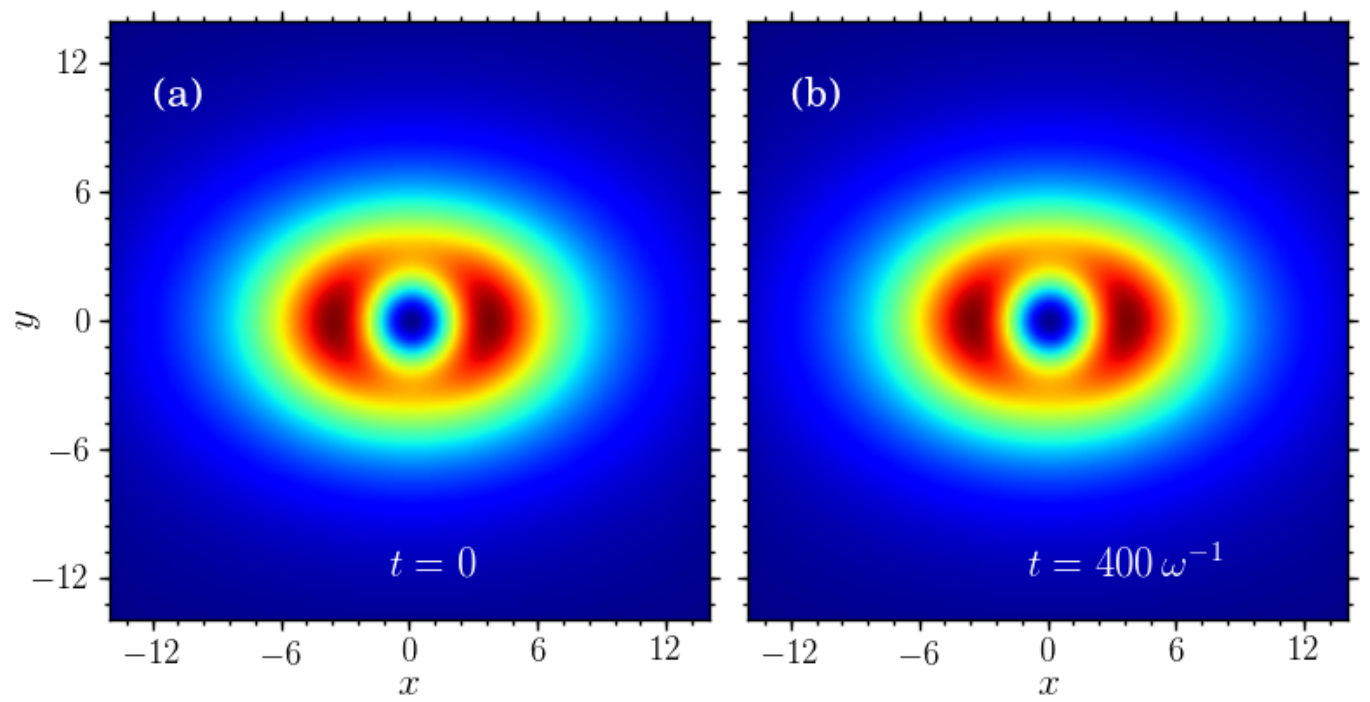

Figure 6. (Color online) The same as in figure 5, but for the elliptic vortex in the anisotropic system with $\epsilon_{1}=0.05$ and $\epsilon_{2}=0.1$. The initial state is produced by the imaginary-time propagation, starting from Gaussian ansatz (7) with $\alpha=0.0403$ and $\gamma=0.7316$ 


\subsection{Vortices with $\ell=2$}

Vortex solitons with the double topological charge are also stable in the presence of the isotropic nonlinear pseudopotential. As shown in figure 7, in the course of long evolution, a perturbation initially added to the isotropic vortex remains trapped in it and causes small persistent irregular perturbations, but does not lead to irreversible fragmentation of the vortex (the evolution picture does not alter, at least, up to $t=2500 \omega^{-1}$, which was the full simulation time).

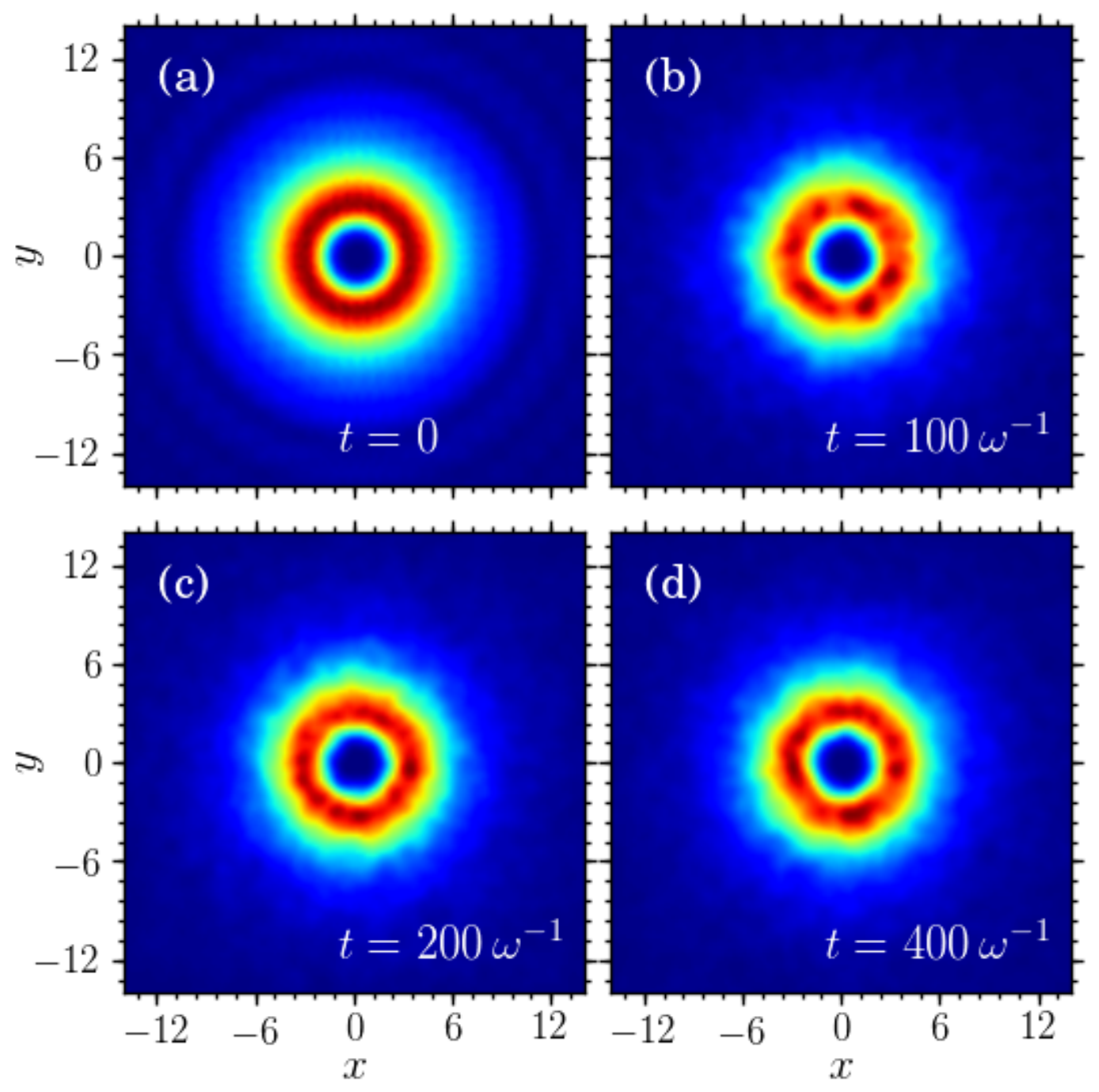

Figure 7. (Color online) The persistent perturbed evolution of the vortex mode with $\ell=2$ under the action of the interactions with strengths $g=20, g_{d}=30$, and the isotropic quartic nonlinear pseudopotential (3) with $\epsilon_{1}=\epsilon_{2}=0.1$.

On the other hand, under the anisotropic quartic pseudopotential, even with a small eccentricity, the double vortex in subject to a splitting instability, as can be seen in figure 8. In fact, the vortical structure is not completely destroyed in this case, but rather splits and recovers periodically. We followed this evolution up to $t=3000 \omega^{-1}$, 

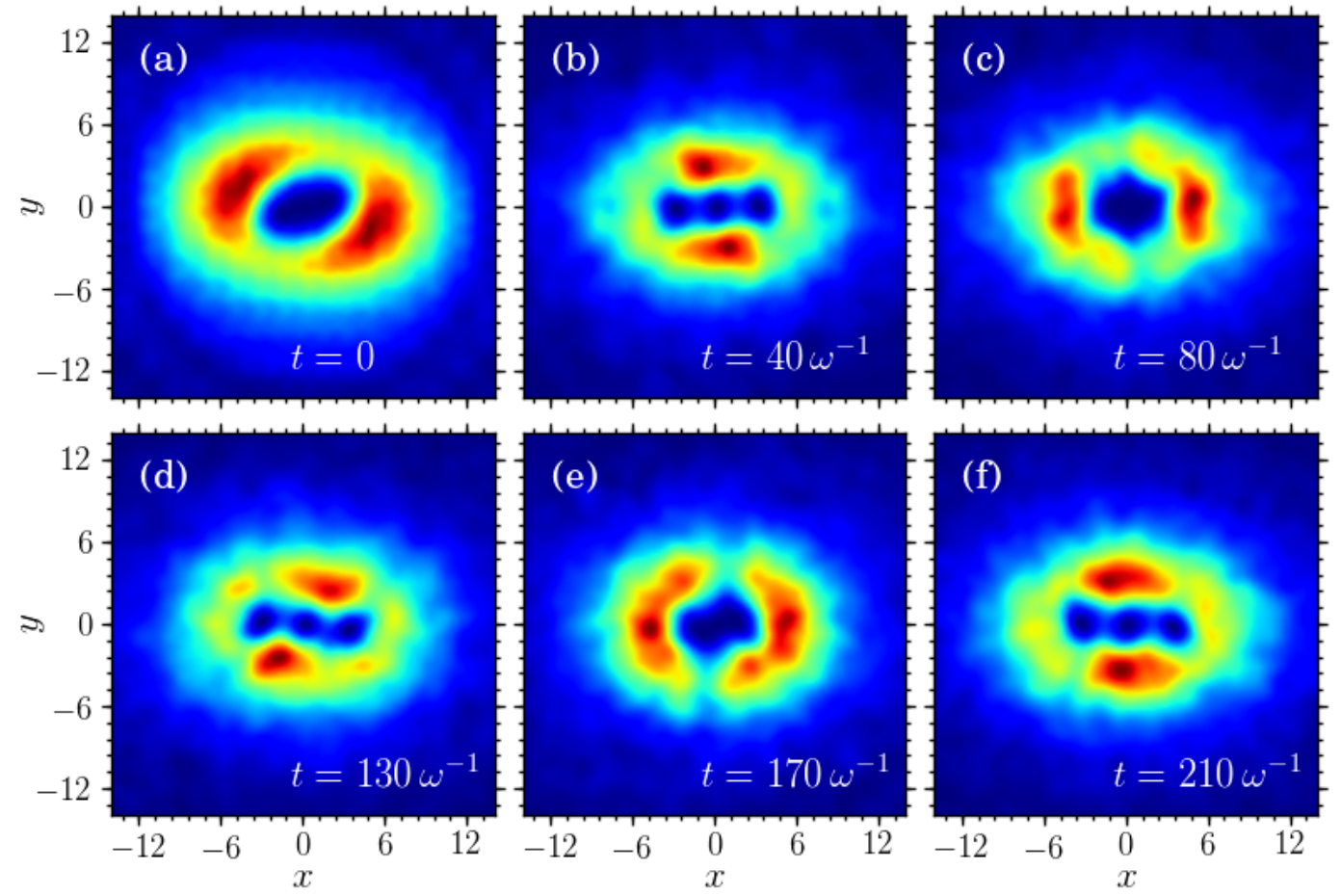

Figure 8. (Color online) The evolution of the elliptic vortex soliton with $\ell=2$ in the dipolar BEC for $g=20, g_{d}=30$, trapped by the anisotropic quartic nonlinear potential (3), with $\epsilon_{1}=0.05, \epsilon_{2}=0.1$.

concluding that the periodic cycles of the splitting and recombination represent a robust dynamical regime. It resembles the known scenario for the evolution of $2 \mathrm{D}$ vortices with $\ell=1$ in the condensate with the uniform attractive contact nonlinearity, trapped in the linear isotropic HO potential, at values of the nonlinearity strength which are intermediate between regions of the full stability and destructive instability (see, e.g., Ref. [31]).

On the basis of systematic simulations, we have concluded that the periodic splitting-recombination regime persists for parameters at which stationary elliptic vortices with $\ell=2$ can be found in the anisotropic pseudopotential. The corresponding period is shown, as a function of eccentricity (4), in figure 9. Naturally, the period decreases with $\delta$, as the transition to the isotropic setting stabilizes the double vortices.

\section{Fundamental solitons $(\ell=0)$}

The VA for the fundamental solitons is based on equations (7)-(14) with $\ell=0$. As it was done above for vortices, numerical solutions have been produced by means of the imaginary-time integration method, starting from the VA prediction. Similar to the situation for the vortical modes, the difference between the VA-predicted and 


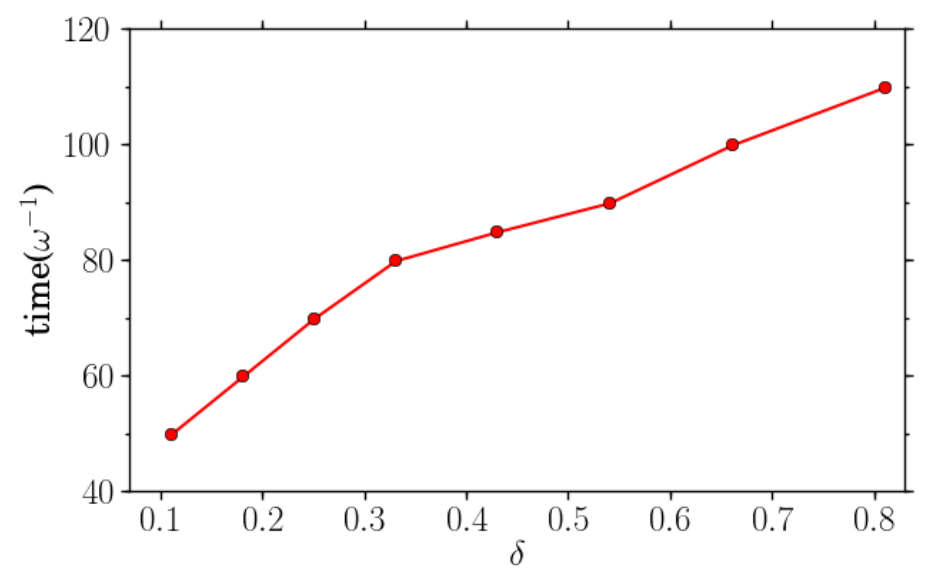

Figure 9. (Color online) The period of the splitting-recombination regime (in units of $\omega^{-1}$ ) for vortices with $\ell=2$, versus the eccentricity $(\delta)$.

numerically generated profiles of the fundamental solitons is $\sim 10 \%$. The characteristics of the family of the fundamental solitons trapped in the isotropic and anisotropic pseudopotentials, similar to those shown for vortices in figures 2 and 3 , are displayed, respectively, in figures 10 and 11 .
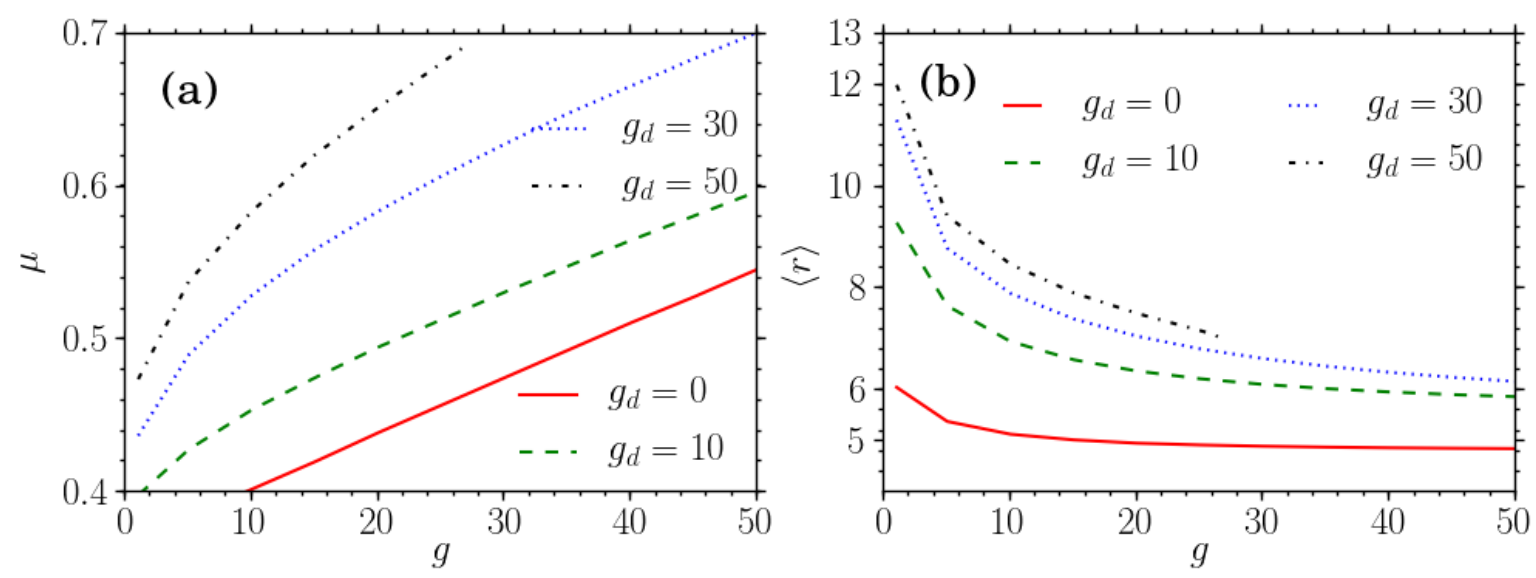

Figure 10. (Color online) The same as in figure 2, but for the fundamental solitons $(\ell=0)$.

As well as the elliptic vortices, elliptic fundamental solitons exist up to a critical (largest) value of the eccentricity. In particular,

$$
\left(\delta_{\mathrm{cr}}\right)_{\ell=0}=0.904
$$

was found at $g=20$ and $g_{\mathrm{d}}=30$, cf. the similar result for the vortices with $\ell=1$, given by equation (15). The critical eccentricity is shown, as a function of the DDI strength, in figure 12, cf. figure 4 for the vortices.

Finally, the fundamental solitons, as well as their counterparts with $\ell=1$, are 

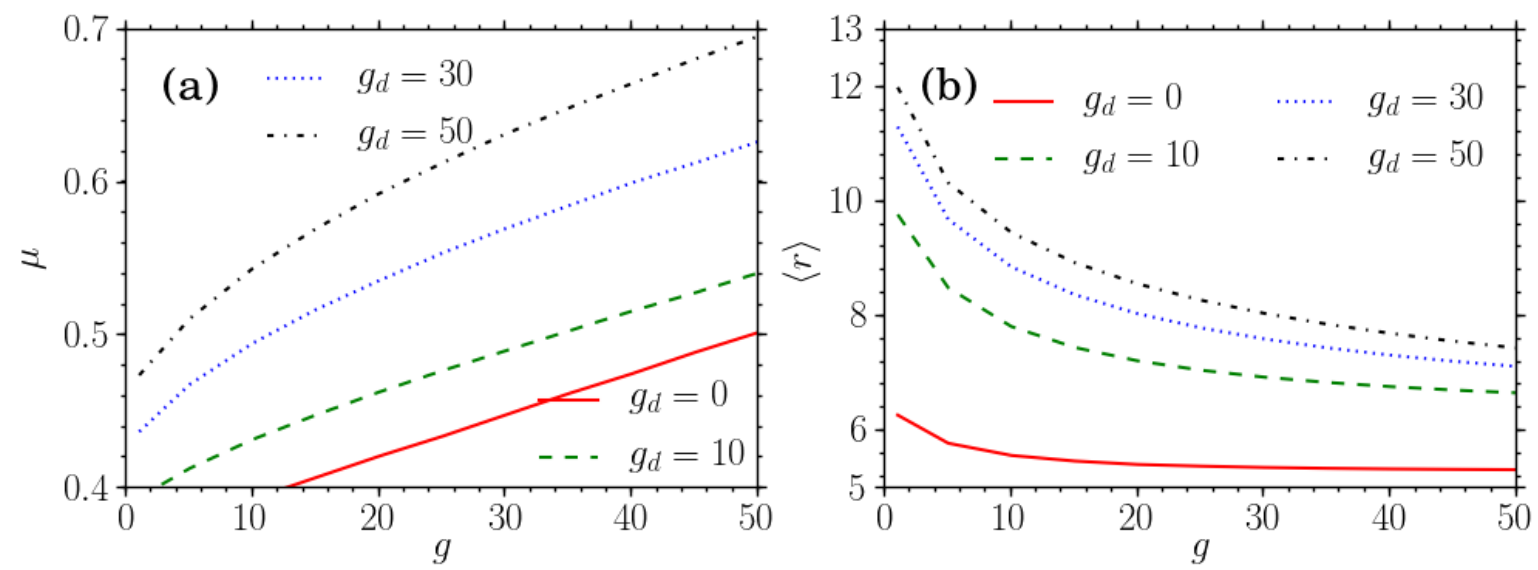

Figure 11. (Color online) The same as in figure 3 , but for elliptic fundamental solitons $(\ell=0)$.

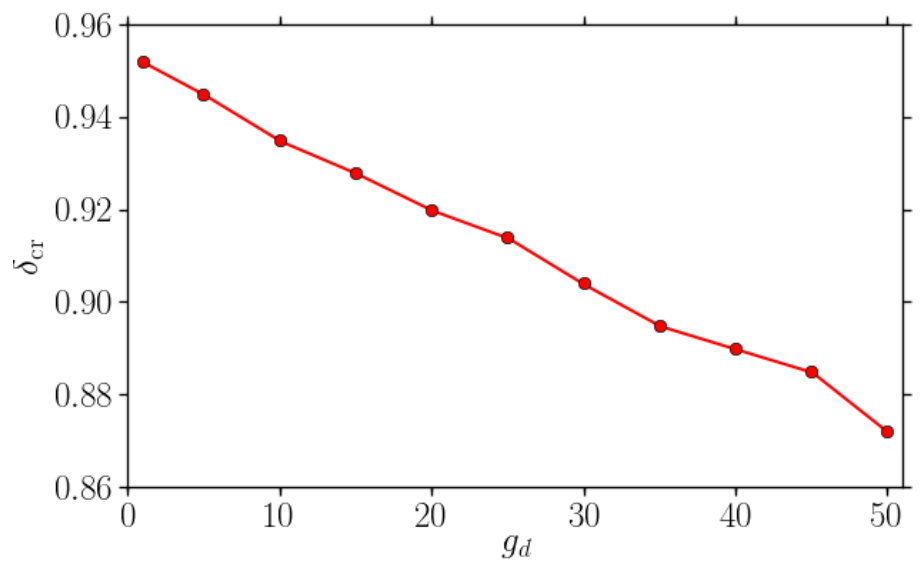

Figure 12. (Color online) The same as in figure 4 but for fundamental solitons $(\ell=0)$.

stable in the isotropic and anisotropic settings alike. The corresponding examples of the stable perturbed evolution are displayed in figures 13 and 14 , respectively.

\section{Conclusion}

The objective of this work is to extend the recently introduced concept of the selftrapping of bright solitons and solitary vortices, supported by the repulsive local nonlinearity with the strength growing from the center to periphery [7], to the case when this trapping mechanism competes with the long-range repulsive DDI (dipoledipole interactions) in the dipolar condensate polarized perpendicular to the plane in which the effective nonlinear potential provides for the self-trapping (the confinement in the perpendicular direction is imposed by the usual linear harmonic-oscillator trap). Another essential extension reported in this paper is the consideration of the self- 

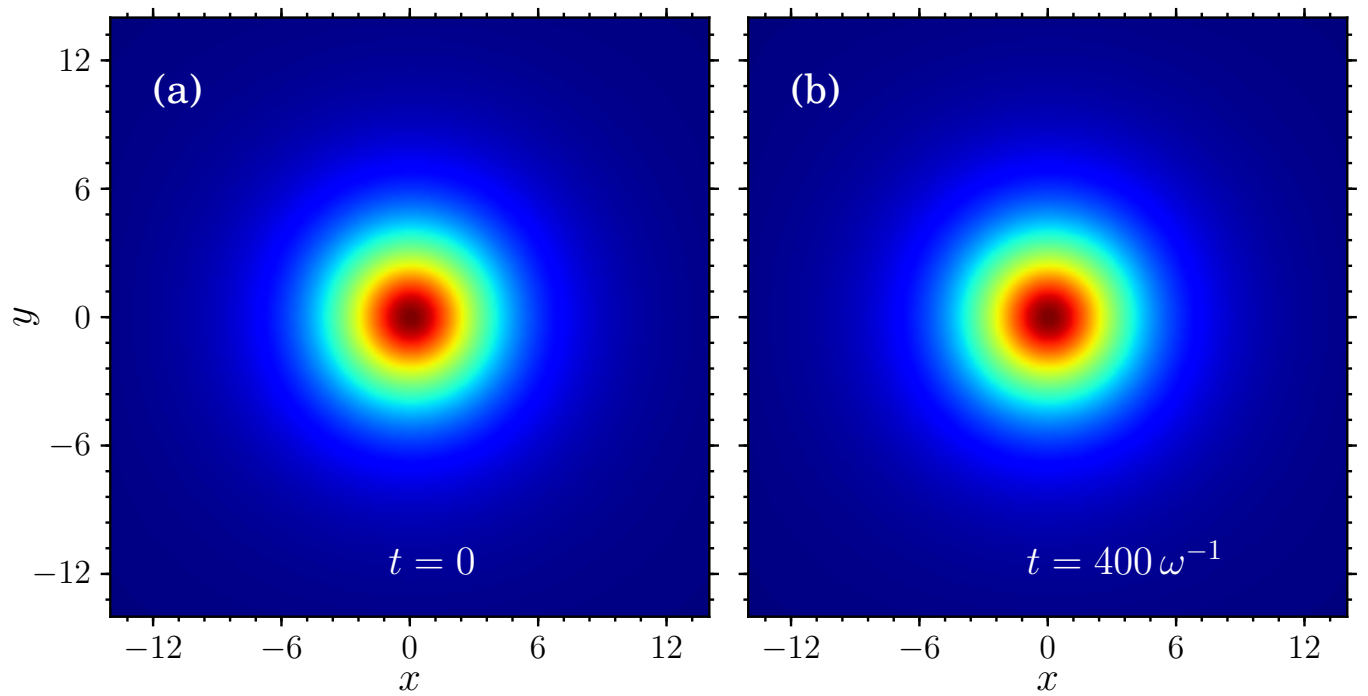

Figure 13. (Color online) The stable evolution of a fundamental soliton, found for the interaction strengths $g=20, g_{d}=30$ and isotropic nonlinearity-modulation profile (3) with $\epsilon_{1}=\epsilon_{2}=0.1$. Shown is the initial state, produced by the imaginary-time propagation starting from Gaussian ansatz (7) with $\ell=0, \alpha=0.0276$ and $\gamma=0.7203$, and the final result of subsequent real-time simulations.
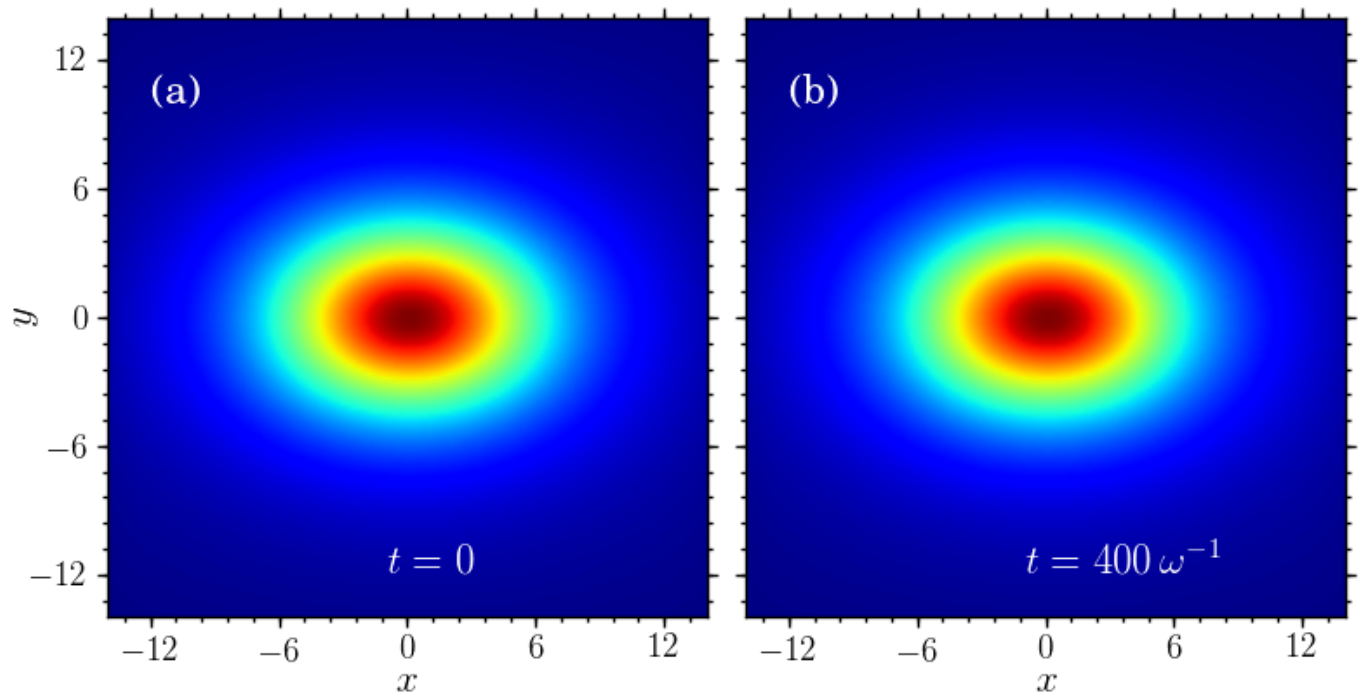

Figure 14. (Color online) The same as in figure 13, but for a stable elliptic fundamental elliptic soliton trapped in the anisotropic pseudopotential with $\epsilon_{1}=0.05$, $\epsilon_{2}=0.1$. The initial state is produced by the imaginary-time propagation starting from Gaussian ansatz (7) with $\alpha=0.0229$ and $\gamma=0.7657$.

trapping of elliptic vortices and fundamental solitons in the anisotropic nonlinear pseudopotential (3). By means of the VA (variational approximation) and numerical simulations in imaginary and real time, we have constructed families of the self-trapped vortical modes with topological charges $\ell=1$ and $\ell=2$, as well as fundamental ones 
with $\ell=0$. It has been found that the fundamental solitons and vortices with $\ell=1$ exist up to critical values of the eccentricity [which are rather close to 1, i.e., they correspond to the strongly elongated elliptic shape, see equations (15) and (16)]. These modes are stable in their entire existence region. On the other hand, the double vortices with $\ell=2$ are stable only under the the circular nonlinear pseudopotential, while the elliptic one triggers an instability mode in the form of periodic breakdown and recovery of the vortical mode.

A challenging possibility for developing the present analysis is to consider a configuration with the in-plane polarization of the dipole moments, which will make the DDI anisotropic, cf. Ref. [20]. An especially intriguing issue is the possibility of the existence of vortical modes in such a setting.

\section{Acknowledgments}

We appreciate valuable discussions with S. K. Adhikari. RKK acknowledges support from the Third World Academy of Sciences (TWAS) and Conselho Nacional de Desenvolvimento Científico e Tecnológico (CNPq, Brazil) for the financial support in the form of a TWAS-CNPq fellowship. The work of PM is a part of research projects funded by the Government of India, through Department of Science and Technology (Ref. No. SR/S2/HEP-03/2009) and Council of Scientific and Industrial Research (Ref. No. 03(1186)/10/EMR-II). BAM appreciates a visitor's grant provided by the South American Institute for Fundamental Research through Instituto de Física Teórica, Universidade Estadual Paulista (São Paulo). The work of this author is also partly supported by the German-Israel Foundation through grant No. I-1024-2.7/2009.

\section{References}

[1] Kivshar Y S and Agrawal G P, Optical Solitons: From Fibers to Photonic Crystals (Academic Press, San Diego, 2003)

[2] Malomed B A, Mihalache D, Wise F and Torner L 2005 J. Optics B: Quant. Semicl. Opt. 7 R53

[3] Lederer F, Stegeman G I, Christodoulides D N, Assanto G, Segev M and Silberberg Y 2008 Phys. Rep. 4631

[4] Yang J, Nonlinear Waves in Integrable and Nonintegrable System (SIAM: Philadelphia, 2010).

[5] Pelinovsky D E, Localization in Periodic Potentials: from Schrödinger Operators to the GrossPitaevskii equations (Cambridge University Press: Cambridge, 2011).

[6] Kartashov Y V, Malomed B A, and Torner L 2011 Rev. Mod. Phys. 83247

[7] Borovkova O V, Kartashov Y V, Malomed B A and Torner L 2011 Opt. Lett. 36 3088-3090 Borovkova O V, Kartashov Y V, Torner L and Malomed B A 2011 Phys. Rev. E 84035602 (R)

[8] Hukriede J, Runde D and Kip D 2003 J. Phys. D 36 R1

[9] Young-S L E, Salasnich L and Malomed B A 2013 Phys. Rev. A 87043603

[10] Inouye S, Andrews M R, Stenger J, Miesner H J, Stamper-Kurn D M and Ketterle W 1998 Nature (London) 392151

Bloch I, Dalibard J and Zwerger W 2008 Rev. Mod. Phys. 80885

Chin C, Grimm R, Julienne P and Tiesinga E ibid. 821225

[11] Fedichev P O, Kagan Y, Shlyapnikov G V and Walraven J T M 1996 Phys. Rev. Lett. 772913 
Theis M, Thalhammer G, Winkler K, Hellwig M, Ruff G, Grimm R and Denschlag J H 2004 ibid. 93123001

Yan M, DeSalvo B J, Ramachandhran B, Pu H and Killian T C 2013 ibid. 110123201

[12] Marinescu M and You L 1998 Phys. Rev. Lett. 814596

[13] Królikowski W, Bang O, Nikolov N I, Neshev D, Wyller J, Rasmussen J J and Edmundson D 2004

J. Opt. B: Quantum Semiclassical Opt. 6 S288

Rotschild C, Cohen O, Manela O, Segev M and Carmon T 2005 Phys. Rev. Lett. 95213904

[14] He Y and Malomed B A 2013 Phys. Rev. A 87053812

[15] Lahaye T, Menotti C, Santos L, Lewenstein M and Pfau T 2009 Rep. Prog. Phys. 72126401

[16] Sinha S and Santos L 2007 Phys. Rev. Lett. 99140406

Lashkin V M 2007 Phys. Rev. A $\mathbf{7 5} 043607$

Nath R, Pedri P and Santos L 2007 ibid. 76013606

Cuevas J, Malomed B A, Kevrekidis P G and Frantzeskakis D J 2009 ibid. 79053608

Nath R, Pedri P and Santos L 2009 Phys. Rev. Lett. 102050401

Cligorić G, Maluckov A, Hadzievski Lj and Malomed B A 2009 J. Phys. B: At. Mol. Opt. Phys.

42145302

Eichler R, Main J and Wunner G 2011 Phys. Rev. A 83053604

Young-S L E, Muruganandam P and Adhikari S K 2011 J. Phys. B: At. Mol. Opt. Phys. 44 101001

Adhikari S K and Muruganandam P 2012 ibid. 45045301

Adhikari S K 2012 Phys. Rev. A 85053631

Li Y, Liu J, Pang W and Malomed B A 2013 ibid. 87013604

[17] Gligorić G, Maluckov A, Hadžievski Lj and Malomed B A 2009 Phys. Rev. A 780636152009 ibid. 79053609

Gligorić G, Maluckov A, Stepić M, Hadžievski Lj and Malomed B A 2010 ibid. 810136332010

J. Phys. B: At. Mol. Opt. Phys. 43055303

Adhikari S K and Muruganandam P 2012 Phys. Lett. A 3762200

[18] Pedri P and Santos L 2005 Phys. Rev. Lett. 95200404

[19] Tikhonenkov I, Malomed B A and Vardi A 2008 Phys. Rev. A 78043614

[20] Tikhonenkov I, Malomed B A and Vardi A 2008 Phys. Rev. Lett. 090406

[21] Köberle P, Zajec D, Wunner G and Malomed B A 2012 Phys. Rev. A 85023630

[22] Baizakov B B, Abdullaev F Kh, Malomed B A, and Salerno M 2009 J. Phys. B: At. Mol. Opt. Phys. 42175302

[23] Muruganandam P and Adhikari S K 2011 J. Phys. B: At. Mol. Opt. Phys. 44121001

[24] Pakter R and Chen C P 2000 Phys. Rev. E 622789

[25] Ghanbari S, Kieu T D, Sidorov A and Hannaford P 2006 J. Phys. B: At. Mol. Opt. Phys. 39847 Abdelrahman A, Hannaford P, and Alameh K 2009 Opt. Express 1724358

[26] Dalfovo F and Stringari S 1996 Phys. Rev. A 532477

Feder D L, Charles W, Clark C W and Schneider B I 1999 Phys. Rev. Lett. 824956

Chiofalo M L, Succi S and Tosi M P 2000 Phys. Rev. E 627438

[27] Svidzinsky A A and Fetter A L 2000 Phys. Rev. Lett. 845919

García-Ripoll J J, Molina-Terriza G, Pérez-García V M and Torner L 2001 ibid. 87140403

García-Ripoll J J and Pérez-García V M 2001 Phys. Rev. A 64013602

[28] Ye F, Dong L, Malomed B A, Mihalache D and Hu B 2010 J. Opt. Soc. Am. B 27757

[29] Muruganandam P and Adhikari S K 2009 Comp. Phys. Commun. 1801888

Vudragović D, Vidanović I, Balaž A, Muruganandam P and Adhikari S K 2012 Comp. Phys.

Commun. 1832021

[30] Sakaguchi H and Malomed B A 2010 Phys. Rev. A 81013624

[31] Mihalache D, Mazilu D, Malomed B A and Lederer F 2006 Phys. Rev. A 73043615 